\title{
Awake craniotomy with transcortical motor evoked potential monitoring for resection of gliomas in the precentral gyrus: utility for predicting motor function
}

\author{
Taiichi Saito, MD, PhD, ${ }^{1}$ Yoshihiro Muragaki, MD, PhD, ${ }^{1,2}$ Manabu Tamura, MD, PhD, ${ }^{2}$ \\ Takashi Maruyama, MD, PhD, ${ }^{1,2}$ Masayuki Nitta, MD, PhD, ${ }^{1}$ Shunsuke Tsuzuki, MD, PhD, ${ }^{1}$ \\ Satoko Fukuchi, MT, ${ }^{3}$ Mana Ohashi, MT, ${ }^{3}$ and Takakazu Kawamata, MD, PhD'
}

${ }^{1}$ Department of Neurosurgery; ${ }^{2}$ Faculty of Advanced Techno-Surgery; and ${ }^{3}$ Central Clinical Laboratory, Tokyo Women's Medical University, Tokyo, Japan

OBJECTIVE Resection of gliomas in the precentral gyrus carries a risk of severe motor dysfunction. To prevent permanent, severe postoperative motor dysfunction, reliable intraoperative predictors of postoperative function are required. Since 2005, the authors have removed gliomas in the precentral gyrus with combined functional mapping and estimation of intraoperative voluntary movement (IVM) during awake craniotomy and transcortical motor evoked potentials (MEPs). The purpose of the current study was to evaluate whether intraoperative findings of combined monitoring of IVM during awake craniotomy and transcortical MEP monitoring were useful for predicting postoperative motor function of patients with gliomas in the precentral gyrus.

METHODS The current study included 30 patients who underwent resection of precentral gyrus gliomas during awake craniotomy from April 2000 to January 2018. All tumors were removed with monitoring of IVM during awake craniotomy and transcortical MEPs. Postoperative motor function was classified as stable or declined, with the extent of decline categorized as mild, moderate, or severe. We defined moderate and severe deficits were those that hindered daily life.

RESULTS In 28 of 30 cases, available waveforms were obtained with transcortical MEPs. The mean extent of resection (EOR) was $93 \%$. Relative to preoperative status, motor function 6 months after surgery was considered stable in 20 patients and was considered to show mild decline in 7, moderate decline in 2, and severe decline in 1. Motor function 6 months after surgery was significantly correlated with IVM $(p=0.0096)$, changes in transcortical MEPs (decline $\leq$ or > $50 \%)(p=0.0163)$, EOR, and ischemic lesions on postoperative MRI. Six patients with no change in IVM showed stable motor function 6 months after surgery. Only 2 patients with a decline in IVM and a decline in MEPs $\leq 50 \%$ had a decline in motor function 6 months after surgery (18\%; $2 / 11$ patients), whereas 11 patients with a decline in IVM and a decline in MEPs > 50\% had such a decline in motor function (73\%; 8/11 patients) including 2 patients with moderate and 1 with severe deficits. Three patients with moderate or severe motor deficits showed the lowest MEP values $(<100 \mu \mathrm{V})$.

CONCLUSIONS Combined judgment from monitoring of IVM during awake craniotomy and transcortical MEPs is useful for predicting postoperative motor function during removal of gliomas in the precentral gyrus. Maximum resection was achieved with an acceptable morbidity rate. Thus, these tumors should not be considered unresectable.

https://thejns.org/doi/abs/10.3171/2018.11.JNS182609

KEYWORDS precentral gyrus; motor function; glioma; awake craniotomy; transcortical motor evoked potentials; oncology

$\mathrm{S}$ URGERY of a glioma in the precentral gyrus carries a risk of critical motor dysfunctions, and thus adequate resection is difficult to achieve. An aggressive surgical strategy has been widely accepted and contributed to a definite improvement in outcomes after treatment for World Health Organization (WHO) grade II, ${ }^{14,22} \mathrm{III},{ }^{3}$ and $\mathrm{IV}^{4,9,21}$ gliomas. On the other hand, glioma patients have a limited life expectancy. Long-lasting neurological deficits associated with surgical removal affect daily life and cause additional distress. To minimize permanent se-

ABBREVIATIONS CMAP = compound muscle action potential; DWI = diffusion-weighted imaging; EOR = extent of resection; IVM = intraoperative voluntary movement MEP = motor evoked potential; WHO = World Health Organization.

SUBMITTED September 8, 2018. ACCEPTED November 27, 2018.

INCLUDE WHEN CITING Published online March 15, 2019; DOI: 10.3171/2018.11.JNS182609. 
vere motor deficits following resection of gliomas from this area, reliable intraoperative predictors of the patient's postoperative motor function are needed.

Cortical and subcortical mapping with electrical stimulation is a useful and reliable method for preserving motor function. ${ }^{1}$ In particular, electrical stimulation for motor mapping during awake craniotomy can simultaneously assess the patient's intraoperative voluntary movement (IVM) in response to verbal commands. ${ }^{25}$ Another method for evaluating the functional continuity of the motor fiber is continuous motor evoked potential (MEP) monitoring. Continuous monitoring of MEPs elicited by direct electrical stimulation with a strip electrode is widely used to improve the safety of removal of gliomas in motor eloquent areas. ${ }^{5,7,20}$ A recent study reported the feasibility and surgical outcome of resection of gliomas in the primary motor cortex..$^{11}$ To preserve motor function and maximize resection, the authors recommended electrical stimulation for mapping of cortical and subcortical motor function. They preferred motor mapping during awake craniotomy. However, they did not use continuous MEP monitoring in conjunction with electrical stimulation motor mapping.

We have removed gliomas in the precentral gyrus with combined functional mapping and estimation of IVM during awake craniotomy and continuous transcortical MEP monitoring since April 2000. The advantage of awake craniotomy with continuous transcortical MEP monitoring is that the surgeon can directly evaluate the association between the change of MEP and IVM of the patient during tumor removal. To the best of our knowledge, no prior studies have reported the application of combined functional mapping and estimation of IVM during awake craniotomy and continuous transcortical MEP monitoring during resection of gliomas in the primary motor area. Thus, we studied 30 consecutive patients with gliomas in the precentral gyrus who underwent an awake craniotomy with transcortical MEP monitoring. The aim of the present study was to evaluate whether intraoperative findings of combined monitoring of IVM during awake craniotomy and continuous transcortical MEP monitoring were useful for predicting postoperative motor function of patients with a glioma in the precentral gyrus.

\section{Methods}

\section{Patients and Preoperative Evaluation}

From April 2000 to January 2018, we performed awake craniotomy in 470 patients at Tokyo Women's Medical University. Of these patients, the current study included 30 consecutive patients with a glioma in the precentral gyrus. We excluded recurrent cases and biopsies performed under general anesthesia. In all cases, the preoperative diagnosis was based on multimodal MRI, which included pre- and postcontrast T1-weighted, T2-weighted, and fluid attenuated inversion recovery images. Using MR images, we evaluated whether the tumors were mainly located in the precentral gyrus. We defined a glioma as being in the precentral gyrus if more than $70 \%$ of the tumor was located in the precentral gyrus. Furthermore, we divided the precentral gyrus into 3 parts, and categorized the tumor location as superior (including leg and shoulder cortex), middle (in- cluding hand cortex), or inferior (including orofacial cortex). The study protocol was approved by the Ethics Committee of the Tokyo Women's Medical University. Because the study is retrospective, the review board did not require informed consent. To protect patient privacy, we removed all identifiers from analyses of the current study.

\section{Surgery}

Surgery was performed according to the previously published concept of information-guided brain tumor removal with the goal of maximal resection of the tumor with minimal risk of permanent postoperative neurological deficits. ${ }^{12,13,16,17}$ The following methods have been published previously. ${ }^{17,18}$ Intraoperative MRI (AIRIS II; Hitachi Medical Corporation) and updated neuronavigation were used routinely. Surgery was generally performed with maximal possible removal of the area defined by enhancement visualized on contrast-enhanced T1-weighted MRI for tumors showing complete or ringed enhancement and suspected to be grade IV gliomas and removal of the area defined by signal hyperintensity on T2-weighted MRI for non- or partially contrast-enhanced tumors suspected to be grade I, II, or III gliomas. Histopathological diagnosis of tumors was based on the previous criteria of WHO in $2007 .{ }^{10}$

\section{Continuous Monitoring of Transcortical MEPs by Direct Cortical Stimulation}

To detect compound muscle action potentials (CMAPs), 27-gauge bipolar disposable subdermal needle electrodes were placed on relevant muscles of the contralateral side of the lesion (abductor pollicis brevis and deltoid for the upper extremity and quadriceps, anterior tibialis, and gastrocnemius for the lower extremity).

After craniotomy and dural opening, a 6-contact titanium strip electrode was placed on the assumed rolandic portion. After placement of the strip electrode, the median nerve was stimulated, and the areas of somatosensory evoked potential (SSEP) phase reversal of the central sulcus and precentral gyrus were identified. Next, cortical motor functional mapping using an Ojemann stimulator was performed as described in the following paragraph. After examining the eloquent cortical areas, we placed the strip electrodes on the margin of the tumor. The position of the strip electrode was adjusted to obtain the maximal CMAPs of target muscles with a threshold of $30 \mathrm{~mA}$ or less. Continuous MEP monitoring by direct cortical stimulation via the strip electrode (train of stimulation, 5; frequency, $500 \mathrm{~Hz}$; pulse duration, $0.5 \mathrm{msec}$ ) was then performed with a neurophysiological monitoring device (Neuromaster MEE-1200; Nihon Kohden) during tumor removal. We defined a reduction in MEP amplitude of more than $50 \%$ as significant and reported this to the surgeon, as described previously. ${ }^{8,24}$

\section{Functional Mapping and Assessment of Voluntary Movement During Awake Craniotomy}

The following methods have been published previously. ${ }^{17}$ In all patients, tumor removal was performed during an awake craniotomy. For intraoperative brain mapping, 
electrical stimulation of the cortex was applied with repetitive square-wave biphasic currents of alternating polarity (pulse width, $0.2 \mathrm{msec}$; frequency, $50 \mathrm{~Hz}$; duration, 1-2 seconds), using an Ojemann cortical stimulator (OCS-1; Integra Radionics, Inc.) and a bipolar electrode with an interpolar distance of $5 \mathrm{~mm}$. The stimulation was performed in a systematic manner along every 8 to $10 \mathrm{~mm}$ of the cortical surface. A continuous digital electrocorticogram was monitored to detect seizures and after-discharges. The stimulus intensity was increased steadily from $2 \mathrm{~mA}$ using stepwise increments of $1 \mathrm{~mA}$ until an effect was attained or electrocorticogram abnormalities were noted. The maximum stimulus intensity was $6 \mathrm{~mA}$ (biphasic current; $12 \mathrm{~mA}$ ). A positive motor response was confirmed by observing whether the relevant muscles moved. The MEP response was obtained by direct cortical stimulation. To confirm reproducible results, each cortical area was stimulated at least 2 times, but never twice in succession. The eloquent cortical areas were delineated with a surgical pen upon completion of mapping. Removal of the tumor was accompanied by subcortical stimulation through the resection cavity, which was intended to identify the motor pathways. The devices used for this purpose and the parameters of stimulation, including the intensity, were similar to those used for cortical mapping. ${ }^{6}$

During the entire procedure while the patient was awake, voluntary movement was monitored by observing the patient's response to verbal commands from a member of the treatment team. In general, the patient's voluntary movement was assessed every 10 minutes, but during removal of the tumor in the vicinity where cortical or subcortical mapping was positive, the motor function was continuously assessed. When the team member confirmed that the patient's voluntary movement had weakened, the degree of weakness was immediately reported to the surgeon. We categorized IVM as showing no change or as declined compared with the preoperative status.

\section{Intraoperative and Postoperative Evaluation}

The tumor resection rate was assessed with intraoperative MRI and postoperative MRI performed within 48 hours after surgery. As in the previously published method, ${ }^{17,18}$ for semiautomatic volumetry to evaluate the extent of resection (EOR) in the present study, we used 3D Slicer 4.0, which is freely downloadable from the website http://www.slicer.org. ${ }^{2}$ For tumors that showed complete or ringed contrast enhancement and were suspected to be grade IV gliomas, we calculated EOR by comparing the contrast-enhanced area on T1-weighted images obtained intraoperatively to that on corresponding images obtained preoperatively; for non- or partially contrast-enhanced tumors suspected to be grade I, II, or III tumors, EOR was calculated based on areas of signal hyperintensity on intra- and preoperative T2-weighted images. To detect ischemic lesions, diffusion-weighted images were also obtained with postoperative MRI studies. We evaluated the patients' motor function on a daily basis until discharge from the hospital. In particular, we categorized motor function 1 week after surgery as declined or stable compared with the preoperative status. Thereafter, all patients were followed regularly in the outpatient clinic by the attending neurosurgeon. We categorized motor function 6 months after surgery as declined or stable compared with the preoperative status. In addition, motor deficits 6 months after surgery were categorized as mild, moderate, or severe as previously reported. ${ }^{11}$ The motor function of each patient was assessed with manual muscle testing (MMT) as previously described. ${ }^{26}$ Mild deficits included MMT grade 4, slight weakness, and slight incoordination, which did not impact daily life. Moderate deficits included MMT grade 2 or 3 with long-term weakness, or a requirement for a brace for walking. Severe deficits included severe motor weakness, MMT grade 0 or 1 , or the presence of contracture.

\section{Statistics}

Data were analyzed using JMP Pro version 14.0 software (SAS Institute). Correlations between motor function 6 months after surgery and various clinical characteristics were analyzed by using Fisher's exact test or an independent t-test. In addition, correlations between the combined findings of IVM and changes in the transcortical MEP response and motor function 1 week or 6 months after surgery were analyzed with Fisher's exact test. Statistical significance was defined as $\mathrm{p}<0.05$.

\section{Results \\ Demographics and Clinical Characteristics}

Representative MR images of 30 patients are shown in Fig. 1. Demographics and clinical data of the 30 patients are shown in Table 1. Approximately half of the tumors $(47 \%)$ were located in the middle part of the precentral gyrus. Only 4 of 30 patients (13\%) presented with preoperative motor weakness. Fourteen patients $(47 \%)$ had preoperative seizures. The mean EOR was $93 \%$ (range $75 \%-$ $100 \%$ ), and the median was $95 \%$. A diffusion-weighted imaging (DWI) study was performed in 25 patients. In the other 5 patients, a DWI study was not performed because these were old cases, and this imaging method was not performed at the time of these operations. Ischemic changes were identified in 10 patients on DWI.

\section{Intraoperative Findings and Postoperative Motor Function}

The intraoperative findings and postoperative motor function of the 30 patients are shown in Table 2. Intraoperative motor mapping was performed in all 30 patients. Electrical stimulation induced muscle movement or an MEP response in the cortical area in $80 \%$ of patients and subcortical fibers in $83 \%$ of patients. The MEP responses in 2 of the 30 patients could not be detected because we could not find a suitable place to obtain an MEP waveform. An intraoperative decline in the MEP of $>50 \%$ was observed in 13 patients (43\%). In 3 of 13 patients with an MEP decline of $>50 \%$, the values recovered to more than $50 \%$ of the control value of the MEP amplitude. A decline in IVM was observed in 25 of 30 patients. Twenty-five patients $(83 \%)$ showed a decline in motor function, including transient weakness, 1 week after surgery compared with the preoperative status, whereas 10 patients (33\%) had a decline in motor function 6 months after surgery compared with the preoperative status. Of these 10 patients, only 3 (10\%; 2 with moderate deficits and 1 with severe deficits) had difficulty in daily life. 

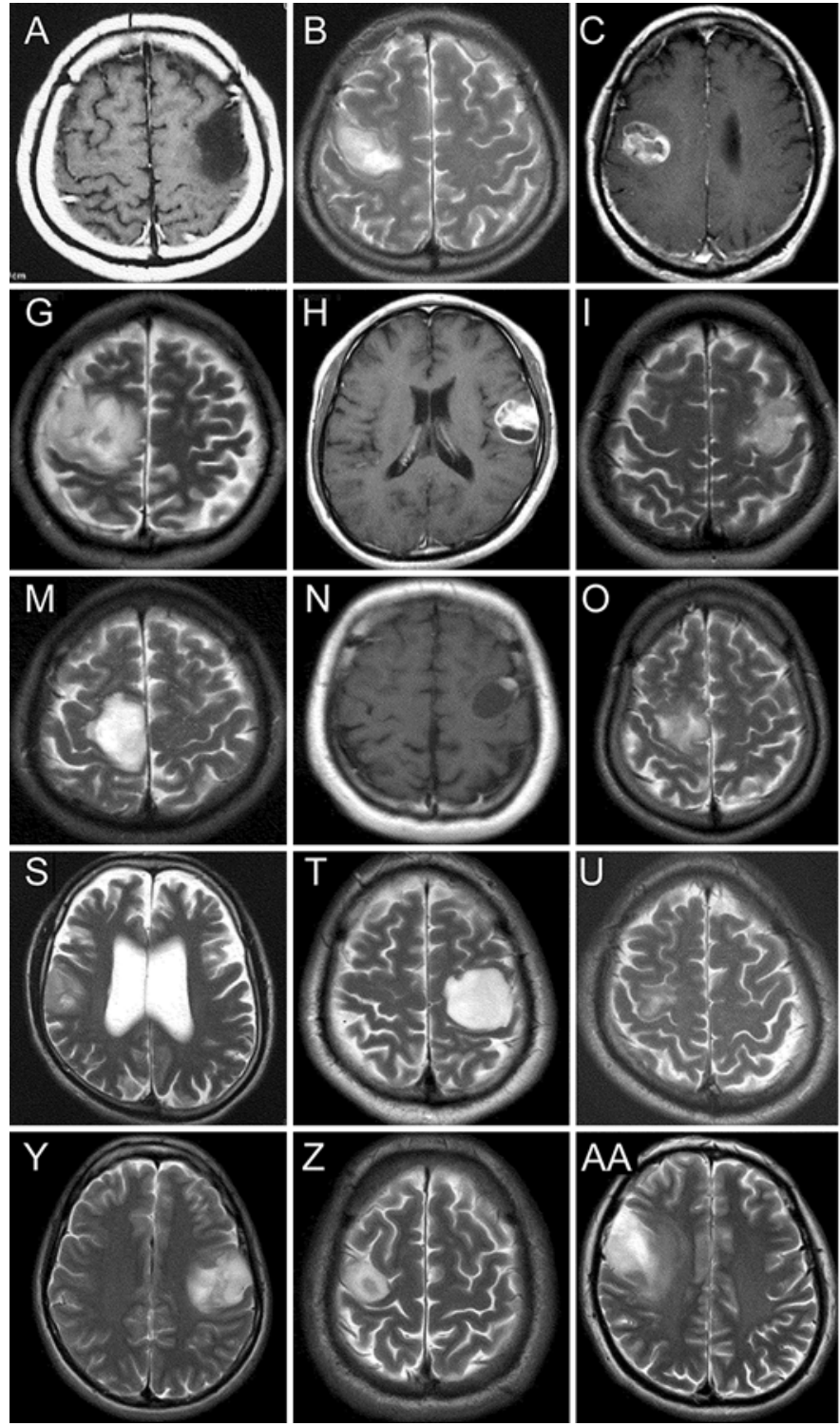
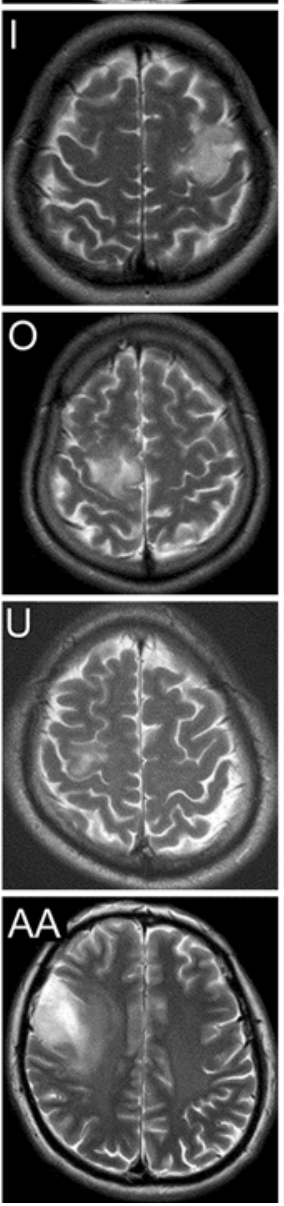
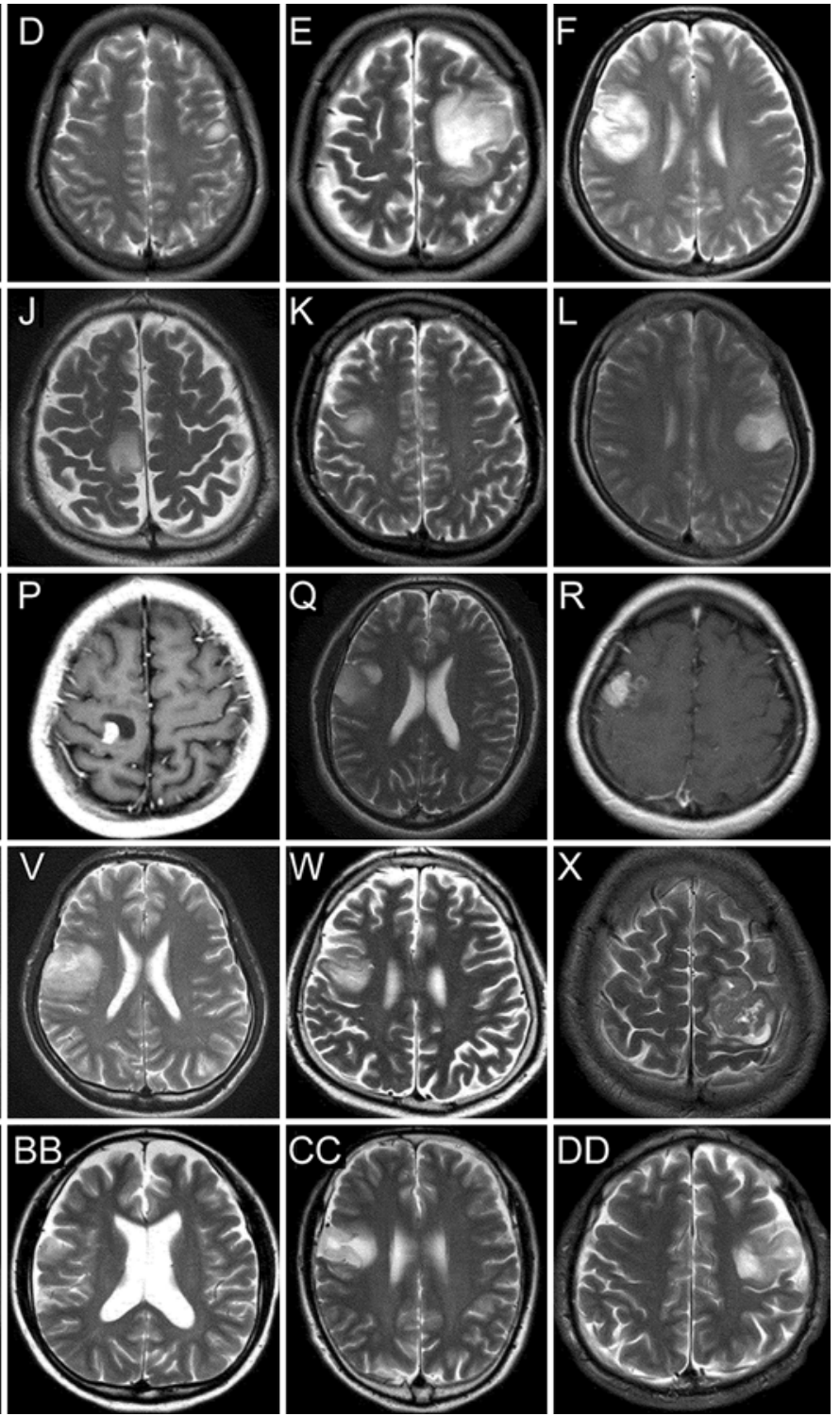

FIG. 1. Key preoperative MR images obtained in 30 patients with gliomas in the precentral gyrus. The key images for cases 1-30 are shown in panels $A-D D$, respectively.

\section{Correlation Between Subgroups of Motor Function 6 Months After Surgery and Clinical Characteristics}

Univariate analyses showed that motor function 6 months after surgery was significantly associated with the EOR $(\mathrm{p}=0.0120)$, ischemic changes on postoperative DWI $(p=0.0113)$, changes in transcortical MEP responses (decline $\leq$ or $>50 \%)(p=0.0163)$, and IVM (no change or declined) $(\mathrm{p}=0.0096)$ (Table 3). No other factors were significantly correlated with motor function 6 months after surgery. The positive and negative predictive values of intraoperative changes in transcortical MEP for discrimination of stable or worsened motor function 6 months after surgery were $62 \%$ and $87 \%$, respectively. In addition, the positive and negative predictive values of intraoperative changes in voluntary movement in discrimination of stable or worsened motor function 6 months after surgery were $43 \%$ and $100 \%$, respectively (Table 3 ).
Correlation Between Motor Function 1 Week or 6 Months After Surgery and IVM Combined With Changes in the Transcortical MEP Response

To predict motor function from the above-mentioned 4 factors, we could confirm only the patient's voluntary movement and changes in the transcortical MEP response during surgery, but not ischemic change or the EOR. Therefore, we evaluated the correlation between motor function 1 week or 6 months after surgery and the combined findings of IVM and changes in transcortical MEPs. The percentage of patients with a decline in motor function is shown in Table 4. Combined findings of IVM (no change or decline) and changes in MEPs (decline in MEPs $\leq 50 \%$ or decline in MEPs $>50 \%$ ) did not correlate with motor function 1 week after surgery $(\mathrm{p}=0.1301)$, but these combined findings were significantly correlated with motor function 6 months after surgery $(\mathrm{p}=0.0090)$. 
TABLE 1. Demographics and clinical data of 30 patients with a glioma in the precentral gyrus

\begin{tabular}{|c|c|}
\hline Variable & Value \\
\hline Total no. of cases & 30 \\
\hline \multicolumn{2}{|l|}{ Sex } \\
\hline Male & 23 \\
\hline Female & 7 \\
\hline \multicolumn{2}{|l|}{ Age, yrs } \\
\hline Mean (range) & $40(23-57)$ \\
\hline Median & 40 \\
\hline \multicolumn{2}{|l|}{ Side of lesion } \\
\hline Left & 11 \\
\hline Right & 19 \\
\hline \multicolumn{2}{|l|}{ Tumor location } \\
\hline Superior & $4(13.3)$ \\
\hline Middle & $14(46.7)$ \\
\hline Inferior & $7(23.3)$ \\
\hline Superior + middle & $2(6.7)$ \\
\hline Middle + inferior & $3(10.0)$ \\
\hline \multicolumn{2}{|c|}{ Preop tumor volume, $\mathrm{cm}^{3}$} \\
\hline Mean (range) & $21.2(1.5-62.0)$ \\
\hline Median & 19 \\
\hline \multicolumn{2}{|l|}{ Preop symptoms } \\
\hline Motor weakness & $4(13.3)$ \\
\hline Seizures & $14(46.7)$ \\
\hline None & $13(43.3)$ \\
\hline \multicolumn{2}{|l|}{ WHO grade } \\
\hline I & $2(6.7)$ \\
\hline II & $18(60.0)$ \\
\hline III & $7(23.3)$ \\
\hline IV & $3(10.0)$ \\
\hline \multicolumn{2}{|l|}{ EOR, \% } \\
\hline Mean (range) & $93(75-100)$ \\
\hline Median & 95 \\
\hline \multicolumn{2}{|c|}{ Ischemic change on postop DWI } \\
\hline Yes & $10(33.3)$ \\
\hline No & $15(50)$ \\
\hline NA & $5(16.7)$ \\
\hline
\end{tabular}

$\mathrm{NA}=$ not applicable.

Values are numbers of cases or patients (\%) unless otherwise indicated.

Six patients with no change in IVM and a decline in MEPs $\leq 50 \%$ or decline in MEPs $>50 \%$ showed no decline in motor function 6 months after surgery. Two patients with a decline in IVM and a decline in MEPs $\leq 50 \%$ had a decline in motor function 6 months after surgery $(18 \%$; 2 of 11 patients). However, these 2 patients had only mild deficits that did not impact daily life (Fig. 2). On the other hand, 8 patients with a decline in IVM and a decline in MEPs $>50 \%$ had a decline in motor function 6 months after surgery (73\%; 8 of 11 patients). These 8 patients included 2 cases with moderate deficits and 1 case with severe deficits (Fig. 2).
TABLE 2. Intraoperative findings and postoperative motor function of 30 patients with a glioma in the precentral gyrus

\begin{tabular}{lc}
\hline \multicolumn{2}{l}{ No. of Cases $(\%)$} \\
\hline Identification of motor-related cortical area \\
\hline Yes & $24(80)$ \\
\hline No & $6(20)$ \\
\hline Identification of motor-related subcortical fibers \\
\hline Yes & $25(83.3)$ \\
\hline No & $5(16.7)$ \\
\hline Transcortical MEP monitoring & \\
\hline Possible & $28(93.3)$ \\
\hline Impossible & $2(6.7)$ \\
\hline Changes in the transcortical MEP response & $15(50.0)$ \\
\hline MEP decline $\leq 50 \%$ & $13(43.3)$ \\
\hline MEP decline $>50 \%$ & $7(23.3)$ \\
\hline Intraop voluntary movement & $23(76.7)$ \\
\hline No change & \\
\hline Declined & $5(16.7)$ \\
\hline Motor function 1 wk postop vs preop status & $25(83.3)$ \\
\hline Stable & $20(66.7)$ \\
\hline Declined & $10(33.3)$ \\
\hline Motor function 6 mos postop vs preop status & $7(23.3)$ \\
\hline Stable & $2(6.7)$ \\
\hline Declined & $1(3.3)$ \\
\hline$\quad$ Mild decline & \\
\hline Moderate decline & \\
\hline Severe decline & \\
\hline & \\
\hline
\end{tabular}

\section{Characteristics of the Patients With a Decline in MEPs $>50 \%$}

Thirteen of 28 patients had a decline in MEPs $>50 \%$. Two of 13 patients showed no change in IVM, whereas 11 patients showed a decline in IVM (Table 5). Two patients with no change in IVM showed stable motor function 6 months after surgery. Of the 11 patients with a decline in IVM, 3 had stable motor function 6 months after surgery (i.e., no decline from preoperative status), 5 had mild deficits, 2 had moderate deficits, and 1 had severe deficits. All 3 patients with moderate and severe deficits showed lowest MEP values of less than $100 \mu \mathrm{V}$ (Table 5, Fig. 2).

\section{Representative Cases}

The descriptions of surgical strategy in this section have been partially published previously. ${ }^{17}$

\section{Case 24}

This case is presented as an example of a case with stable IVM and a decline in MEPs $\leq 50 \%$.

A 23-year-old man experienced generalized seizures. He had no other neurological symptoms. The tumor showed signal isointensity and hyperintensity on T2-weighted images (Fig. 3A). Tumor removal was performed during an awake craniotomy. Electrical stimulations identified muscle contraction of the right hand and lower extremity. We removed the tumor with guidance from cortical and sub- 
TABLE 3. Correlation between subgroups of motor function 6 months after surgery and clinical characteristics of 30 patients with a glioma in the precentral gyrus

\begin{tabular}{|c|c|c|c|}
\hline \multirow[b]{2}{*}{ Characteristic } & \multicolumn{2}{|c|}{$\begin{array}{l}\text { 6-Mo Postop vs } \\
\text { Preop Status }\end{array}$} & \multirow{2}{*}{$\begin{array}{l}\text { Univariate } \\
\text { p Value }\end{array}$} \\
\hline & Stable & Declined & \\
\hline No. of pts & 20 & 10 & \\
\hline \multicolumn{4}{|l|}{ Sex } \\
\hline Male & 14 & 9 & 0.1976 \\
\hline Female & 6 & 1 & \\
\hline Age (mean), yrs & 38.0 & 42.7 & 0.2288 \\
\hline Side of lesion & & & 0.7894 \\
\hline Left & 7 & 4 & \\
\hline Right & 13 & 6 & \\
\hline Tumor location & & & 0.0547 \\
\hline Superior & 1 & 3 & \\
\hline Middle & 10 & 4 & \\
\hline Inferior & 6 & 1 & \\
\hline Superior + middle & 0 & 2 & \\
\hline Middle + inferior & 3 & 0 & \\
\hline Preop tumor volume, $\mathrm{cm}^{3}$ & 17.9 & 27.9 & 0.0919 \\
\hline WHO grade & & & 0.1263 \\
\hline I & 2 & 0 & \\
\hline II & 12 & 6 & \\
\hline III & 3 & 4 & \\
\hline IV & 3 & 0 & \\
\hline EOR, \% & 95.5 & 88.0 & 0.0120 \\
\hline Ischemic change on postop DWI & & & 0.0113 \\
\hline Yes & 3 & 7 & \\
\hline No & 12 & 3 & \\
\hline Preop motor weakness & & & 0.4587 \\
\hline Yes & 2 & 2 & \\
\hline No & 18 & 8 & \\
\hline $\begin{array}{l}\text { Identification of motor-related cortical } \\
\text { area }\end{array}$ & & & 0.3432 \\
\hline Yes & 17 & 7 & \\
\hline No & 3 & 3 & \\
\hline $\begin{array}{l}\text { Identification of motor-related subcor- } \\
\text { tical fibers }\end{array}$ & & & 0.6573 \\
\hline Yes & 16 & 7 & \\
\hline No & 4 & 3 & \\
\hline $\begin{array}{l}\text { Changes in transcortical MEP } \\
\text { response }\end{array}$ & & & 0.0163 \\
\hline MEP decline $\leq 50 \%$ & 13 & 2 & \\
\hline MEP decline $>50 \%$ & 5 & 8 & \\
\hline IVM & & & 0.0096 \\
\hline No change & 7 & 0 & \\
\hline Declined & 13 & 10 & \\
\hline
\end{tabular}

Values are numbers of patients or cases unless otherwise indicated. Statistical analysis was performed with Fisher's exact test and an independent t-test. cortical stimulation for motor mapping with constant monitoring of the patient's voluntary movement. We confirmed the motor response of the right hand and lower-extremity muscle on the white matter. During removal of the tumor, no motor dysfunction was noted. Intraoperative monitoring of transcortical MEPs showed no decline (abductor pollicis brevis, $1630 \mu \mathrm{V}$ to $1770 \mu \mathrm{V}$ ) (Fig. 3B). Postoperative MRI revealed total resection of the tumor (Fig. 3C). The pathological diagnosis was pleomorphic xanthoastrocytoma. The patient had no motor weakness 1 week or 6 months after surgery.

\section{Case 12}

This case is presented as an example of a case with a decline in IVM and a decline in MEPs $\leq 50 \%$.

A 31-year-old woman presented with right facial partial seizures. She had no other neurological symptoms. MRI showed a tumor centered in the inferior part of the left precentral gyrus (Fig. 3D). The tumor showed signal hyperintensity on T2-weighted images and showed no enhancement on T1-weighted images obtained after administration of contrast medium. The tumor was removed during an awake craniotomy. Electrical stimulations identified right facial muscle contraction. We removed the tumor with guidance from cortical and subcortical stimulation for motor mapping with constant monitoring of the patient's voluntary movement. We could not confirm motor response on the white matter. During removal of the tumor, mild motor dysfunction of the right finger was noted. The intraoperative transcortical MEPs showed no decline (abductor pollicis brevis, $4100 \mu \mathrm{V}$ to $3970 \mu \mathrm{V}$ ) (Fig. 3E). Postoperative MRI revealed $95 \%$ resection of the tumor (Fig. 3F). The pathological diagnosis was oligodendroglioma. The patient had mild motor weakness 1 week after surgery, but her motor function had recovered at 6 months after surgery.

\section{Case 10}

This case is presented as an example of a case with a decline in IVM and a decline in MEPs $>50 \%$.

A 49-year-old man presented with generalized seizures. $\mathrm{He}$ had no other neurological symptoms. MRI showed a tumor centered in the superior part of the right precentral gyrus (Fig. 3G). The tumor showed signal hyperintensity on T2-weighted images and showed no enhancement on T1-weighted images obtained after administration of contrast medium. Tumor removal was performed during an awake craniotomy. Electrical stimulations identified left finger muscle contraction, but not contraction of the lower-extremity muscles. We removed the tumor with guidance from subcortical stimulation for motor mapping with constant monitoring of the patient's voluntary movement. We confirmed motor responses of the left anterior tibialis and gastrocnemius muscle on the white matter. During removal of the tumor, severe motor dysfunction of the left lower extremity was noted, and an obvious decrease was seen in transcortical MEPs (gastrocnemius, $600 \mu \mathrm{V}$ to 25 $\mu \mathrm{V})$ (Fig. 3H, Table 5). Postoperative MRI revealed 85\% resection of the tumor (Fig. 3I). The pathological diagnosis was oligodendroglioma. The patient had severe motor weakness 1 week after surgery and severe motor deficits 6 months after surgery. 
TABLE 4. Correlation between motor function 1 week or 6 months after surgery and intraoperative observations of presence or absence of change in voluntary movement combined with transcortical MEP response in 30 patients with a glioma in the precentral gyrus

\begin{tabular}{|c|c|c|c|c|c|c|}
\hline \multirow[b]{2}{*}{ Intraop Observations } & \multicolumn{3}{|c|}{ 1-Wk Postop Motor Function } & \multicolumn{3}{|c|}{ 6-Mo Postop Motor Function } \\
\hline & No Change & Declined & $\%$ of Cases w/ Decline & Stable & Declined & $\%$ of Cases w/ Decline \\
\hline \multicolumn{7}{|c|}{ No change in voluntary movement } \\
\hline Decline in MEPs $\leq 50 \%$ & 2 & 2 & $50 \%$ & 4 & 0 & $0 \%$ \\
\hline Decline in MEPs $>50 \%$ & 1 & 1 & $50 \%$ & 2 & 0 & $0 \%$ \\
\hline \multicolumn{7}{|l|}{ Decline in voluntary movement } \\
\hline Decline in MEPs $\leq 50 \%$ & 1 & 9 & $90 \%$ & 9 & 2 & $18 \%$ \\
\hline Decline in MEPs $>50 \%$ & 1 & 11 & $92 \%$ & 3 & 8 & $73 \%$ \\
\hline$p$ value & & & 0.1301 & & & 0.0090 \\
\hline
\end{tabular}

Values are cases of patients unless otherwise indicated. Statistical analysis was performed with Fisher's exact test.

\section{Discussion}

\section{Main Results}

Results of the current study suggest that the combination of intraoperative monitoring of changes in the patient's voluntary movement and transcortical MEPs is useful for predicting motor function 6 months after surgery in patients with a glioma in the precentral gyrus. Motor function 6 months after surgery was significantly associated with IVM, changes in the transcortical MEP response, EOR, and ischemic change on DWI. Additionally, combined findings of IVM and changes in transcortical MEPs were well correlated with motor function 6 months after surgery. Notably, 6 patients with no change in IVM showed stable motor function 6 months after surgery regardless of whether they showed a decline in MEPs $\leq 50 \%$ or not, whereas the 22 patients with a decline in IVM included 10 patients with a decline in motor function. In addition, 11 patients (73\%) with a decline in IVM and a decline in MEPs $>50 \%$ showed motor deficits 6 months after surgery, including 2 patients with moderate deficits and 1 patient with severe deficits. Ten patients with a decline in motor function 6 months after surgery had a significantly lower EOR than the patients who had stable motor function. This result is

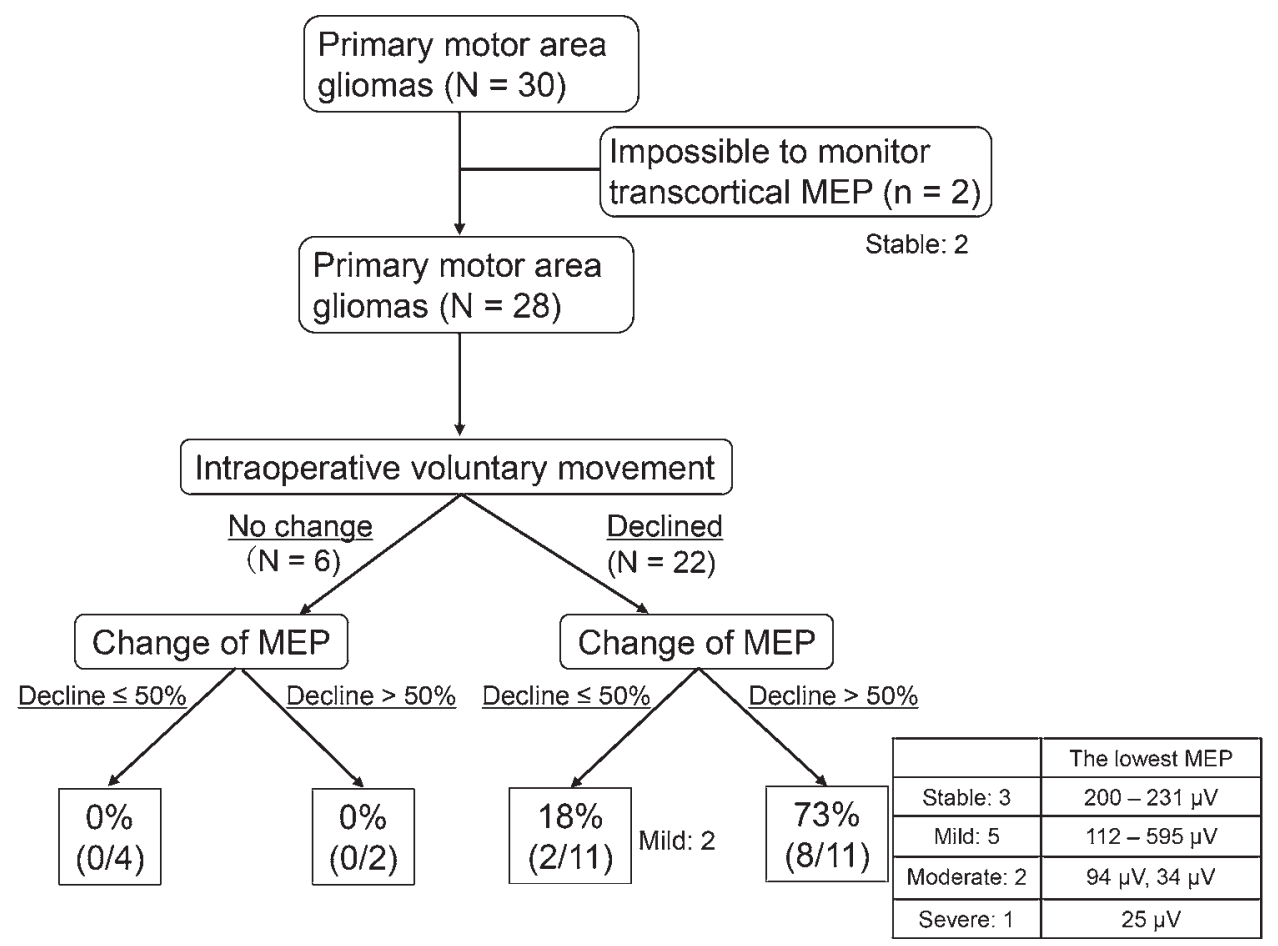

The rate of declined cases in motor function 6 months after surgery

FIG. 2. Flowchart showing cases in which the combination of IVM monitoring during awake craniotomy and transcortical MEP monitoring was used and the correlation between findings on intraoperative monitoring and the percentage of cases in which patients showed a decline in motor function 6 months after surgery. MEP monitoring was performed by direct cortical stimulation via the strip electrode (train of stimulation, 5 ; frequency, $500 \mathrm{~Hz}$; pulse duration, $0.5 \mathrm{msec}$ ). 
TABLE 5. Characteristics of 13 patients with an MEP decline $>50 \%$

\begin{tabular}{|c|c|c|c|c|c|c|c|c|}
\hline & Age & Sex & Side & $\begin{array}{l}\text { WHO } \\
\text { Grade }\end{array}$ & $\begin{array}{c}\text { Ischemic } \\
\text { Changes on } \\
\text { Postop DWI }\end{array}$ & $\begin{array}{l}\text { Control } \\
\text { Value of } \\
\text { MEPs }\end{array}$ & $\begin{array}{l}\text { Lowest } \\
\text { MEP Value }\end{array}$ & $\begin{array}{l}\text { 6-Mo Postop } \\
\text { Motor Function }\end{array}$ \\
\hline \multicolumn{9}{|c|}{$\begin{array}{l}\text { No change in intraop voluntary move- } \\
\text { ment }\end{array}$} \\
\hline Case 14 & 40 & M & Lt & I & No & $1018 \mu \mathrm{V}$ & $0 \mu \mathrm{V}$ & Stable \\
\hline Case 23 & 39 & M & Rt & II & No & $2700 \mu \mathrm{V}$ & $0 \mu \mathrm{V}$ & Stable \\
\hline \multicolumn{9}{|c|}{ Decline in intraop voluntary movement } \\
\hline Case 2 & 37 & M & Rt & II & NA & $1092 \mu \mathrm{V}$ & $200 \mu \mathrm{V}$ & Stable \\
\hline Case 3 & 57 & M & Rt & IV & NA & $776 \mu \mathrm{V}$ & $231 \mu \mathrm{V}$ & Stable \\
\hline Case 7 & 33 & M & Rt & III & Yes & $404 \mu \mathrm{V}$ & $94 \mu \mathrm{V}$ & Mod def \\
\hline Case 9 & 52 & $\mathrm{~F}$ & Lt & II & No & $1297 \mu \mathrm{V}$ & $595 \mu \mathrm{V}$ & Mild def \\
\hline Case 10 & 49 & M & Rt & II & Yes & $600 \mu \mathrm{V}$ & $25 \mu \mathrm{V}$ & Severe def \\
\hline Case 13 & 44 & $M$ & $\mathrm{Rt}$ & II & No & $2708 \mu \mathrm{V}$ & $34 \mu \mathrm{V}$ & Mod def \\
\hline Case 15 & 30 & M & Rt & II & Yes & $1391 \mu \mathrm{V}$ & $232 \mu \mathrm{V}$ & Mild def \\
\hline Case 20 & 45 & M & Lt & III & No & $3320 \mu \mathrm{V}$ & $320 \mu \mathrm{V}$ & Mild def \\
\hline Case 22 & 32 & M & Rt & II & Yes & $4960 \mu \mathrm{V}$ & $620 \mu \mathrm{V}$ & Mild def \\
\hline Case 27 & 39 & M & Rt & III & No & $2050 \mu \mathrm{V}$ & $200 \mu \mathrm{V}$ & Stable \\
\hline Case 30 & 44 & $M$ & Lt & II & Yes & $487 \mu \mathrm{V}$ & $199 \mu \mathrm{V}$ & Mild def \\
\hline
\end{tabular}

due to interruption of tumor resection because of a decline in MEPs $>50 \%$. The significant correlation between ischemic changes on DWI and motor function in the present study is compatible with findings of prior retrospective cohort studies..$^{5,8}$

\section{Feasibility of Resection of Gliomas in the Precentral Gyrus}

Although many neurosurgeons assume that gliomas in the precentral gyrus are unresectable, we have resected gliomas in this area with the combination of awake craniotomy and continuous transcortical MEP monitoring. We have been able to achieve maximum resection of gliomas in this area with a mean EOR of $93 \%$ and median of $95 \%$. Of 30 patients, only $3(10 \%)$ had motor deficits that impacted daily life (severe deficits in 1 case and moderate deficits in 2 cases). A recently published study reported the feasibility and surgical outcomes of 53 resections of gliomas within the motor cortex. ${ }^{11}$ The authors describe resection with electrical stimulation mapping of cortical and subcortical motor function. Awake craniotomy was performed in $65 \%$ of cases, whereas $35 \%$ were done under general anesthesia. The mean EOR was $91 \%$. Of the patients with permanent motor deficits, only $6(11 \%)$ showed motor deficits that impacted daily living. The authors report surgical outcomes comparable to ours, although their operations were performed without continuous MEP monitoring with motor mapping. On the other hand, another published study reported surgical results in 29 cases of malignant glioma in the primary sensorimotor cortex. ${ }^{15}$ In those cases tumor resection was performed with continuous transcranial MEP monitoring, electrical stimulation for motor mapping, and 5-aminolevulinic acid fluorescence guidance without awake craniotomy. The authors reported that a deterioration in motor function was seen in only $16 \%$ of patients. These surgical outcomes suggest the feasibility of resection of gliomas in the precentral gyrus with an acceptable morbidity rate and confirm the validity of our surgical strategy. The novelty in the present study is that we used the combined results of monitoring of IVM during awake craniotomy and transcortical MEPs to predict postoperative motor function of patients with gliomas in the precentral gyrus.

\section{Surgical Benefits of Combined Awake Craniotomy and Transcortical MEP Monitoring in Predicting Motor Func- tion}

We believe that the surgical strategy of combined awake craniotomy and continuous transcortical MEP monitoring has a lower risk of permanent motor deficits. The most useful advantage of combined awake craniotomy with continuous transcortical MEP monitoring is that the surgeon can directly observe IVM of the patient during tumor removal while comparing changes in MEP results. In the present study, as a first step, we found that the 6 patients with no change in IVM showed stable motor function 6 months after surgery, whereas of 22 patients with a decline in IVM, 10 had a decline in motor function 6 months after surgery (Table 4, Fig. 2). Of the 6 patients with no change in IVM, 2 patients showed a decline in MEPs $>50 \%$, but these patients had stable motor function 6 months after surgery (Fig. 2). In these patients, MEP changes indicated false-positive results (Table 5). Presumably, as previously reported, ${ }^{23}$ shifting of the strip electrode on the cortical surface due to brain shift underneath the electrode can lead to inaccurate recordings not directly caused by intraoperative impairment of motor function. In a prior study, 

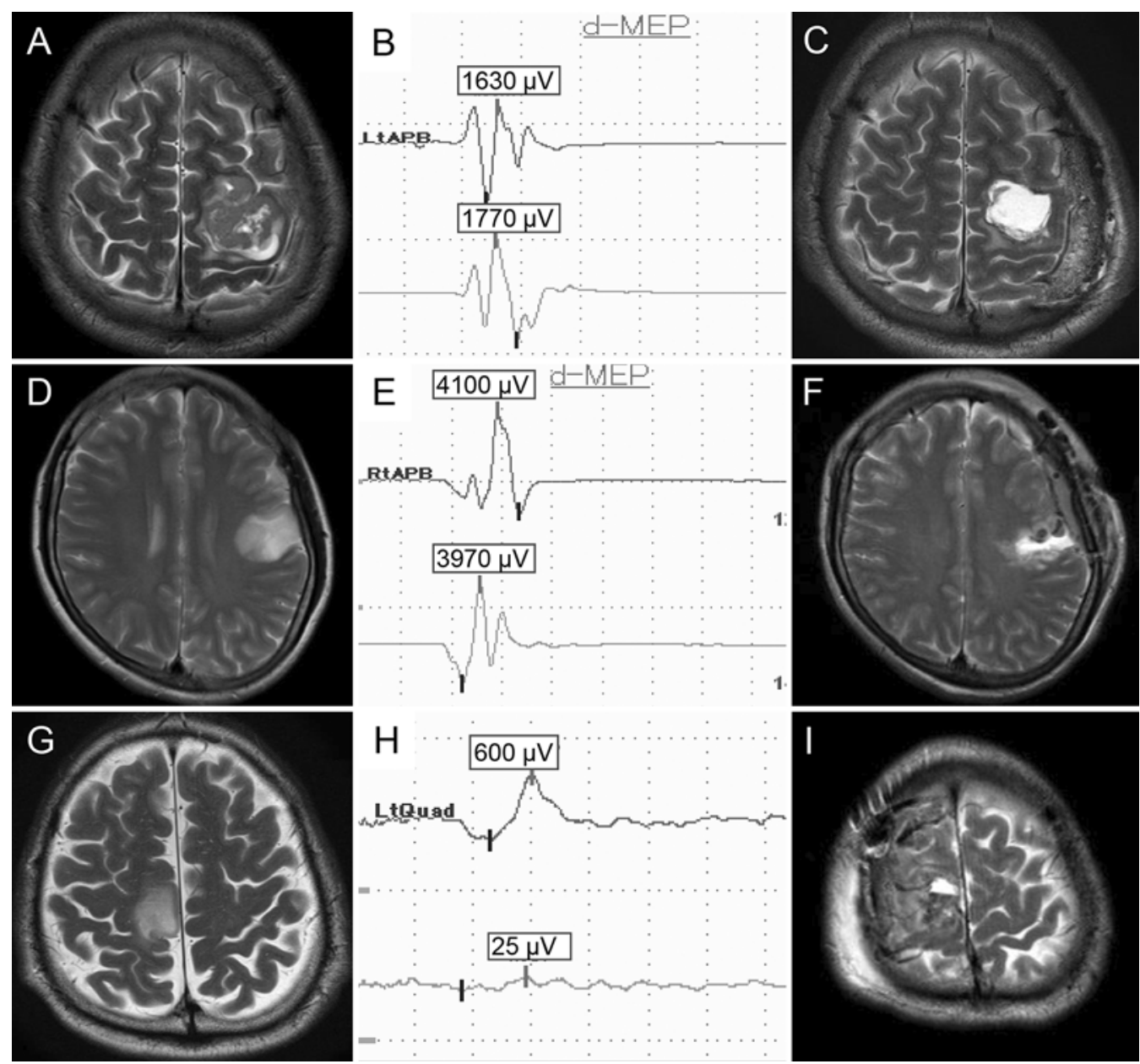

FIG. 3. Three representative cases of a glioma in the primary motor area. A-C: Case 24; example of a case with a stable IVM and a decline in MEPs $\leq 50 \%$. Preoperative T2-weighted MRI (axial image in A) revealed a tumor with isointense and hyperintense signal in the superior and middle portion of the left precentral gyrus. Changes in transcortical MEPs were observed during surgery (B; upper: control, lower: during tumor removal). Postoperative MRI (axial T2-weighted image in C) revealed total resection of the tumor. D-F: Case 12; example of a case with a decline in IVM and a decline in MEPs $\leq 50 \%$. Preoperative T2-weighted MRI (axial image in D) revealed a high-signal intensity mass centered in the inferior part of the left precentral gyrus. Changes in transcortical MEPs were observed during surgery (E; upper: control, lower: during tumor removal). Postoperative MRI (axial T2-weighted image in F) revealed $95 \%$ resection of the tumor. G-I: Case 10; example of a case with a decline in IVM and a decline in MEPs > $50 \%$. Preoperative T2-weighted MRI (axial image in G) revealed a high-signal intensity mass in the superior portion of the right precentral gyrus. Changes were observed in transcortical MEPs during surgery $(\mathrm{H}$; upper: control; lower: the lowest MEP during tumor removal). Postoperative MRI (axial T2-weighted image in I) revealed $85 \%$ resection of the tumor. APB $=$ abductor pollicis brevis; d-MEP = direct MEP; Quad = quadriceps.

3 of 31 cases $(9.7 \%)$ showed a significant change in the recorded motor responses due to intraoperative dislocation of the stimulating electrode. ${ }^{23}$ In the second step, of 22 patients with a decline in IVM, 11 patients showed a decline in MEPs $\leq 50 \%$. Only 2 of these 11 patients had mild motor deficits, which did not affect daily life (Fig. 2). In contrast, 11 of 22 patients with a decline in IVM showed a decline in MEPs $>50 \%$. Of these 11 patients, $8(73 \%)$ had motor dysfunction 6 months after surgery including 2 patients with moderate deficits and 1 patient with severe deficits. These results suggest a complementary relationship between monitoring of voluntary movement during awake craniotomy and transcortical MEPs. The combination of these 2 factors is useful for removal of gliomas in the precentral gyrus and for predicting postoperative motor function.

\section{Threshold of Transcortical MEPs for Predicting Moderate or Severe Motor Dysfunction}

In the current study, we defined a reduction in MEP amplitude of 50\% as significant, as reported in prior studies. ${ }^{8,24}$ However, of 11 patients with a decline in IVM and a decline in MEPs $>50 \%, 3$ patients had stable motor function and 5 patients had mild motor dysfunction 6 months after surgery (Table 5). The daily life of these 8 patients was not affected by their motor function. From a clinical point of view, identification of the threshold of MEPs for predicting moderate or severe motor dysfunction that impacts the daily life of patients is important. We have empirically considered that less than $100 \mu \mathrm{V}$ for MEPs is a valuable criterion for predicting permanent severe motor dysfunction, as reported in 2 previous review articles. ${ }^{16,19}$ Indeed, 2 patients with moderate deficits and 1 patient with 
severe deficits showed lowest MEP values of less than 100 $\mu \mathrm{V}$, whereas 8 patients with stable or mild motor dysfunction showed lowest MEP values of more than $100 \mu \mathrm{V}$ (Fig. 2 , Table 5). These results suggest that tumor removal may be tolerated as long as the MEPs are maintained at values greater than $100 \mu \mathrm{V}$, at least in our monitoring.

\section{Limitations}

The present study has some limitations. First, our study was retrospective in nature. Therefore, despite the rather promising results presented here, additional prospective studies are needed. Second, the current study was performed at a single institution, and therefore, further multicenter studies are required to generalize our surgical strategy. Third, we did not include recurrent cases in this study. To further validate our surgical strategy, additional investigations including recurrent cases are needed.

\section{Conclusions}

We demonstrated that a complementary relationship is present between the capacity for voluntary movement during awake craniotomy and transcortical MEPs, and the combined evaluation of these 2 factors is useful for removal of gliomas in the precentral gyrus and predicting motor function 6 months after surgery. Combined results from changes in IVM and transcortical MEPs were well correlated with motor function 6 months after surgery. We could achieve maximum resection of these tumors with an acceptable morbidity rate, and thus, these tumors should not be considered unresectable. Additionally, the value of $100 \mu \mathrm{V}$ could be the threshold of transcortical MEPs for predicting moderate or severe motor dysfunction 6 months after surgery.

\section{Acknowledgments}

This study was supported by the Japan Society for the Promotion of Science (JSPS) KAKENHI Grant No. 18K09006. We are especially grateful to Dr. Takashi Komori, Ms. Soko Ikuta, Asuka Komori, and Mr. Takashi Sakayori for valuable help with clinical work and data analysis.

\section{References}

1. De Witt Hamer PC, Robles SG, Zwinderman AH, Duffau H, Berger MS: Impact of intraoperative stimulation brain mapping on glioma surgery outcome: a meta-analysis. J Clin Oncol 30:2559-2565, 2012

2. Egger J, Kapur T, Fedorov A, Pieper S, Miller JV, Veeraraghavan $\mathrm{H}$, et al: GBM volumetry using the 3D Slicer medical image computing platform. Sci Rep 3:1364, 2013

3. Fujii Y, Muragaki Y, Maruyama T, Nitta M, Saito T, Ikuta S, et al: Threshold of the extent of resection for WHO Grade III gliomas: retrospective volumetric analysis of 122 cases using intraoperative MRI. J Neurosurg 129:1-9, 2018

4. Fukui A, Muragaki Y, Saito T, Maruyama T, Nitta M, Ikuta $\mathrm{S}$, et al: Volumetric analysis using low-field intraoperative magnetic resonance imaging for 168 newly diagnosed supratentorial glioblastomas: effects of extent of resection and residual tumor volume on survival and recurrence. World Neurosurg 98:73-80, 2017

5. Gempt J, Krieg SM, Hüttinger S, Buchmann N, Ryang YM, Shiban E, et al: Postoperative ischemic changes after glioma resection identified by diffusion-weighted magnetic reso- nance imaging and their association with intraoperative motor evoked potentials. J Neurosurg 119:829-836, 2013

6. Kayama T: The guidelines for awake craniotomy: Guidelines Committee of the Japan Awake Surgery Conference. Neurol Med Chir (Tokyo) 52:119-141, 2012

7. Kombos T, Picht T, Derdilopoulos A, Suess O: Impact of intraoperative neurophysiological monitoring on surgery of high-grade gliomas. J Clin Neurophysiol 26:422-425, 2009

8. Krieg SM, Shiban E, Droese D, Gempt J, Buchmann N, Pape $\mathrm{H}$, et al: Predictive value and safety of intraoperative neurophysiological monitoring with motor evoked potentials in glioma surgery. Neurosurgery 70:1060-1071, 2012

9. Lacroix M, Abi-Said D, Fourney DR, Gokaslan ZL, Shi W, DeMonte F, et al: A multivariate analysis of 416 patients with glioblastoma multiforme: prognosis, extent of resection, and survival. J Neurosurg 95:190-198, 2001

10. Louis DN, Ohgaki H, Wiestler OD, Cavenee WK, Burger PC, Jouvet A, et al: The 2007 WHO classification of tumours of the central nervous system. Acta Neuropathol 114:97-109, 2007

11. Magill ST, Han SJ, Li J, Berger MS: Resection of primary motor cortex tumors: feasibility and surgical outcomes. J Neurosurg 129:961-972, 2018

12. Muragaki Y, Iseki H, Maruyama T, Kawamata T, Yamane F, Nakamura R, et al: Usefulness of intraoperative magnetic resonance imaging for glioma surgery. Acta Neurochir Suppl 98:67-75, 2006

13. Muragaki Y, Iseki H, Maruyama T, Tanaka M, Shinohara C, Suzuki T, et al: Information-guided surgical management of gliomas using low-field-strength intraoperative MRI. Acta Neurochir Suppl 109:67-72, 2011

14. Nitta M, Muragaki Y, Maruyama T, Ikuta S, Komori T, Maebayashi K, et al: Proposed therapeutic strategy for adult low-grade glioma based on aggressive tumor resection. Neurosurg Focus 38(1):E7, 2015

15. Noell S, Feigl GC, Naros G, Barking S, Tatagiba M, Ritz R: Experiences in surgery of primary malignant brain tumours in the primary sensori-motor cortex practical recommendations and results of a single institution. Clin Neurol Neurosurg 136:41-50, 2015

16. Saito T, Muragaki Y, Maruyama T, Tamura M, Nitta M, Okada Y: Intraoperative functional mapping and monitoring during glioma surgery. Neurol Med Chir (Tokyo) 55:1-13, 2015

17. Saito T, Muragaki Y, Maruyama T, Tamura M, Nitta M, Tsuzuki S, et al: Difficulty in identification of the frontal language area in patients with dominant frontal gliomas that involve the pars triangularis. J Neurosurg 125:803-811, 2016

18. Saito T, Muragaki Y, Tamura M, Maruyama T, Nitta M, Tsuzuki S, et al: Impact of connectivity between the pars triangularis and orbitalis on identifying the frontal language area in patients with dominant frontal gliomas. Neurosurg Rev [epub ahead of print], 2018

19. Saito T, Tamura M, Chernov MF, Ikuta S, Muragaki Y, Maruyama T: Neurophysiological monitoring and awake craniotomy for resection of intracranial gliomas. Prog Neurol Surg 30:117-158, 2018

20. Saito T, Tamura M, Muragaki Y, Maruyama T, Kubota Y, Fukuchi S, et al: Intraoperative cortico-cortical evoked potentials for the evaluation of language function during brain tumor resection: initial experience with 13 cases. J Neurosurg 121:827-838, 2014

21. Sanai N, Mirzadeh Z, Berger MS: Functional outcome after language mapping for glioma resection. $\mathbf{N}$ Engl J Med 358:18-27, 2008

22. Smith JS, Chang EF, Lamborn KR, Chang SM, Prados MD, Cha S, et al: Role of extent of resection in the long-term outcome of low-grade hemispheric gliomas. J Clin Oncol 26:1338-1345, 2008 
23. Suess O, Kombos T, Ciklatekerlio O, Stendel R, Suess S, Brock M: Impact of brain shift on intraoperative neurophysiological monitoring with cortical strip electrodes. Acta Neurochir (Wien) 144:1279-1289, 2002

24. Suess O, Suess S, Brock M, Kombos T: Intraoperative electrocortical stimulation of Brodman area 4: a 10-year analysis of 255 cases. Head Face Med 2:20, 2006

25. Szelényi A, Bello L, Duffau H, Fava E, Feigl GC, Galanda $\mathrm{M}$, et al: Intraoperative electrical stimulation in awake craniotomy: methodological aspects of current practice. Neurosurg Focus 28(2):E7, 2010

26. Takakura T, Muragaki Y, Tamura M, Maruyama T, Nitta M, Niki C, et al: Navigated transcranial magnetic stimulation for glioma removal: prognostic value in motor function recovery from postsurgical neurological deficits. J Neurosurg 127:877-891, 2017

\section{Disclosures}

The authors report no conflict of interest concerning the materi- als or methods used in this study or the findings specified in this paper.

\section{Author Contributions}

Conception and design: Muragaki, Saito. Acquisition of data: Saito, Tamura, Maruyama, Nitta, Tsuzuki, Fukuchi, Ohashi. Analysis and interpretation of data: Muragaki, Saito, Tamura, Tsuzuki, Fukuchi, Ohashi. Drafting the article: Saito. Critically revising the article: Muragaki, Saito, Kawamata. Reviewed submitted version of manuscript: Saito, Maruyama, Nitta, Kawamata. Approved the final version of the manuscript on behalf of all authors: Muragaki. Statistical analysis: Saito. Administrative/technical/material support: Tamura, Maruyama, Nitta, Tsuzuki, Fukuchi, Ohashi. Study supervision: Kawamata.

\section{Correspondence}

Yoshihiro Muragaki: Tokyo Women's Medical University, Tokyo, Japan.ymuragaki@twmu.ac.jp. 